\title{
OPTIMAL PLANNING AND OPERATION OF ACTUAL DISTRIBUTION SYSTEMS IN EGYPT USING A MULTI-OBJECTIVE GENETIC ALGORITHM
}

\author{
Adel A. Abou El-Ela \\ Faculty of Engineering \\ Minoufiya University
}

\author{
Gamal E. M. Aly \\ Faculty of Engineering \\ Tanta University
}

\author{
Abeer A. E. Shammah \\ South Delta Electricity \\ Distribution Company SDEDC
}

\begin{abstract}
This paper proposes procedures for optimal planning and operation of radial distribution systems (RDS). Optimal planning involves selection of the best cross-section of branches to be operated, such that the resulting RDS yields the desired performance for the existing and expanding load demand. A genetic algorithm (GA) is used to obtain the optimal branches of RDS by minimizing the sum of branch cost and present worth of the feeder energy costs, while keeping the voltage regulation within a prescribed limit and satisfying the growth factor. The multi-objective genetic algorithm (MOGA) is suggested to achieve more than one objective and satisfy the RDS constraints. After selection of the optimal branches, an optimal operation procedure of RDS is proposed using a distribution system software programming (DSSP). This program is applied to obtain the optimal switched point to find the best radial reconfiguration system with minimum energy loss costs and satisfy the RDS conistraints such as: branch voltage drop, thermal current carrying capacity and balanced power generation-load demand equation. Different studies are presented to illustrate the capability of the proposed optimal procedures using two real life power distribution systems.

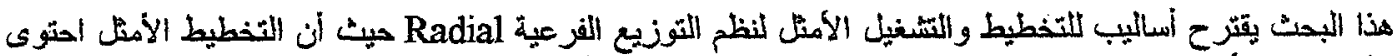

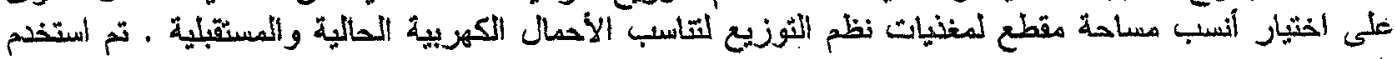

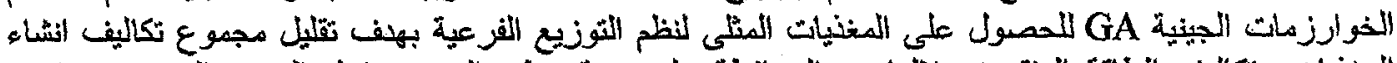

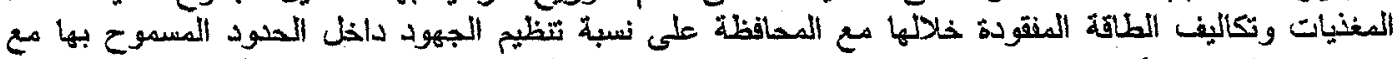

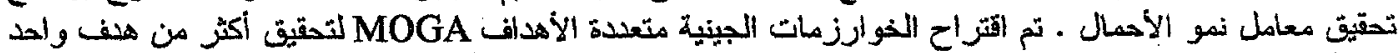

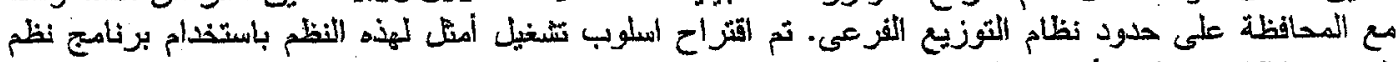

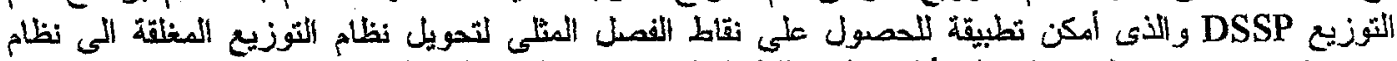

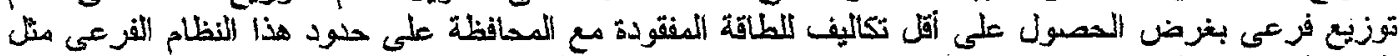

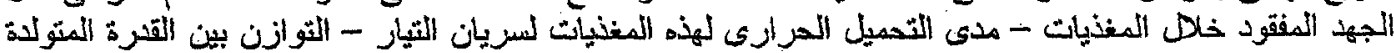

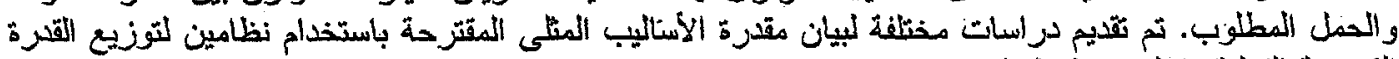
الكهربية الفعلية داخل مدينة طنطا.
\end{abstract}

Keywords: Distribution system, Genetic Algorithm, Multi-objective genetic algorithm.

\section{INTRODUCTION}

Planning and operation of distribution systems involve a long list of optimization problems, like expansion at minimum cost or network reconfiguration keeping in mind a certain objective function (e.g., feeder and/or substation balancing, loss reduction, voltage profile, etc.).

A key factor when practically implementing those optimization problems refers to the fact that, while distribution networks are structurally meshed, they are radially operated. The optimal planning and operation of distribution networks are achieved when the networks present minimum losses, minimum voltage deviations at the customer loading points and maximum reliability. Network reconfiguration is the process of altering the topological structures of distribution feeders by changing the open/closed states of the sectionalizing and tie switches under both normal and abnormal operating conditions. During normal operating conditions, networks are reconfigured to reduce the system real power losses, relieve loads in the network and to increase network reliability. When a fault occurs on a feeder, the 
faulted section has to be identified and isolated. The isolated sections will have to be fed from alternative feeders until the faulty branch is repaired. Several categories of network reconfiguration techniques for power loss reduction can be distinguished [1]. On the one hand, the safest way to solve the reconfiguration problem consists of trying all possible trees for the system at hand, as done in [2]. On the other hand, the combinatorial nature of the problem has held researchers to explore purely heuristic solution techniques [3-5].

Finally, a third category comprises those contributions using artificial intelligence, like simulated annealing [6], fuzzy logic[7], genetic algorithms [8] (whose computation speed is too slow to apply to large systems), or artificial neural networks[9] (which requires substational amount of accurate data for training). Ref. [10] presented an optimization methodology for distribution networks expansion using an evolutionary algorithm. This methodology deals only with planning distribution problems. Ref. [11] presented a systematic procedure to study the effect of temperature change to the power system load demand (load profiles and feeder losses). Recently, reconfiguration of distribution system considering loss minimization were presented in $[12,13]$.

This paper presents a proposed procedure to obtain the optimal cross-section branches to be suitable for existing and extending distribution networks, while the other procedure deals with the operation of RDS for normal and abnormal operating conditions.

\section{PROBLEM FORMULATION}

Planning and operation of distribution systems involve a multi-objective functions that must be achieved while necessary system constraints are satisfied.

\subsection{Objective Functions}

a. Minimization of energy loss costs:

This paper aims to minimize the system power loss which can be expressed as:

$$
M i n F_{1}=\operatorname{Min} \sum_{i=1}^{n} 3 T 10^{-3} I_{i}^{2} R_{i}(L L F)
$$

where, $\mathrm{n}$ is the total number of branches, Ii is the load current flow in branch $i, \mathrm{Ri}$ is the resistance of branch $i, T$ is the number of operating hours per year and LLF is the loss of load factor which is a function of load factor (LF) and is defined as [14]:

$$
L L F=A(L F)^{2}+B(L F) \text { where, } A+B=1
$$

The total energy loss is to be calculated on the basis of present worth cost for the period of conductor, assumed life time (D years) for a discount rate of annual percentage $r$. Therefore, the energy loss costs can be expressed as:

$$
\operatorname{MinF}=\sum_{i=1}^{n} 3 T 10^{-3} I^{2} R_{i}(L L F) h \sum_{d=1}^{D}\left(1 /(1+r)^{d}\right)
$$

where, $h$ is the cost of energy per KWH.

\section{b. Minimization of feeder costs:}

The actual cost of the distribution feeder involves a fixed cost as well as a variable cost. The fixed cost component relates to the pole and involves, labor and installation. The variable cost component reflects the cost of conductor material and is a function of crosssectional area. Then, the minimization of total costs over the life period of the feeders in the distribution system can be expressed as:

$\operatorname{MinF}_{2}=\sum_{k=1}^{D} \sum_{i=1}^{n}\left(w_{1 i} a_{i}+w_{2 i}\right) l_{i}$

where, $W_{l i}$ and $W_{2 i}$ are the cost constants of feeder $i$ per unit length $(1 \mathrm{~km})$.

\section{c. Minimization of voltage deviation:}

The voltage deviation with respect to the flat voltage (1.0 p.u.) must be minimized, so that the regulation factor at load bus in the distribution system can be modified. This objective function can be expressed as:

$$
\operatorname{MinF}_{3}=\sum_{j=1}^{N}\left(V_{j}-V^{s p}\right)
$$

where, $V_{j}$ is the voltage magnitude at bus $j, V^{\infty p}$ is specified voltage (1.0p.u) and $N$ is the total number of load buses.

\subsection{Constraints of distribution system:}

The minimization of the objective functions (3)-(5) may results in cross-sectional areas that may lead to either high or low service quality.

a-Voltage level constraints:

The Voltage level at the consumer, in the distribution system, is the main constraint. The voltage drop in the distribution feeder depends on the choice of its cross-section area, loading level, power factor and circuit operating voltage. However, the small value of feeder voltage drop leads to high conductor size and, consequently, more investment and lower system losses. The voltage magnitude at each bus must be maintained within its limits as:

$$
V_{j}^{\min } \leq V_{j} \leq V_{j}^{\max } \quad \mathrm{j}=1, \ldots, \mathrm{N}
$$

where, $\mathrm{V}_{j}^{\min }$ and $\mathrm{V} j^{\max }$ are the minimum and maximum voltage magnitude at bus $j$.

\section{b. Current flow constraint:}

This current has to lie within its capacity rating of branch $\mathbf{j}$ as follow: 


$$
\left|I_{j}\right| \leq I_{j}^{\max }
$$

where, $\left|I_{j}\right|$ and $I_{j}^{\max }$ are the current magnitude and maximum current limit of branch $j$, respectively.

\section{c. Thermal-limit constraint:}

The maximum allowable conductor temperature, at which the conductor can be operated, is called the thermal limit or thermal rating of that conductor. This constraint can be expressed as:

$$
A_{j} \geq A^{\min }
$$

where, $A_{j}$ and $A^{\text {min }}$ are cross-section area and its minimum limit of branch $j$. However, the minimum cross-section area in the first branch must carry the total current of ring distribution before transferring it to RDS.

\section{d. Balanced equation:}

The total current flow must be equal to the total nodal loading current according to the following equation:

$$
\sum_{i=1}^{n} I_{i}=\sum_{j=1}^{N} I L_{j}
$$

where, $I L_{j}$ is loading current at node $j$.

\section{GENETIC ALGORITHM FOR OPTIMAL CROSS-SECTION AREA OF BRANCHES}

Genetic algorithms (GAs) are based on the mechanisms of natural selection. GAs produce high quality solutions because they are independent of the choice of initial configurations. Initial population is generated randomly. Population consists of individuals, each representing a particular selection of the values of the variables coded in binary form. Each individual is evaluated to obtain a measure of its fitness in terms of objective function to be optimized.

In this paper, GA is used to find the optimal crosssection area of branches to achieve multi objective functions (3)-(5). After this start, successive population are generated using the following three basic operators:

(1) Reproduction

(2) Crossover

(3) Mutation

1) Reproduction Operator:

Reproduction is a process in which individual strings are copied according to their objective function value (fitness). In this process, strings with higher value have a higher probability of contributing one or more off-springs to the next generation.
2) Crossover Operator:

After reproduction, crossover is used to create new individuals and takes place according to a given probability value as shown in Fig. 1 .

3) Mutation Operator:

Mutation creates a new individual by changing a randomly selected bit in its coding as shown in Fig. 2.

\begin{tabular}{|l|l|l|l|l|}
\hline 0 & 1 & 1 & 1 & 0 \\
\hline 1 & 1 & 0 & 1 & 1 \\
\hline
\end{tabular}

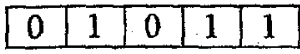

(a)Before crossover

(b)After crossover Fig.1 Crossover operator

\begin{tabular}{|l|l|l|l|l|}
\hline 0 & 1 & 0 & 1 & 0 \\
\hline
\end{tabular}$\quad$\begin{tabular}{|l|l|l|l|l|}
\hline 0 & 1 & 1 & 1 & 0 \\
\hline
\end{tabular}

Fig. 2 Mutation Operator

\section{MULTI-OBJECTIVE GENETIC ALGORITHM (MOGA)}

Many real-world problems involve simulation and optimization of several objective functions. Generally, these functions often have competing and conflicting objectives. Multi-objective optimization with such conflicting objective functions gives rise to sets of optimal solutions, instead of one optimal solution. The reason for the optimality of many solutions is that no one can be considered to be better than any other with respect to all objective functions. A general multi-objective optimization problem consists of one or more objectives to be optimized simultaneously and is associated with a number of constraints. It can be formulated as follows:

$$
\begin{aligned}
& \quad \operatorname{Min} f_{i}(x) \quad i=1, \ldots, N o \\
& \text { subject to } \begin{cases}g_{j}(x) \geq 0 & j=1, \ldots, M \\
h_{k}(x) \leq 0 & k=1, \ldots, k_{c}\end{cases}
\end{aligned}
$$

where, is the ith objective functions, $x$ is a decision vector that represents a solution, $\mathrm{N} 0$ is the number of objective functions and $g_{f}(x) \& h_{k}(x)$ are inequality constraints of power system.

The priority goal programming (PGP) is used to achieve the simultaneous optimization of several objective functions. These objective functions can be expressed as:

$$
\operatorname{Min} f=w_{1} \cdot F_{1}+w_{2} \cdot F_{2}+w_{3} \cdot F_{3}
$$

Since, there is no feasible solution when these objectives are minimized simultaneously, the priority goal programming technique must be used. This technique is very efficiently to be used dependent on the selection of the best values of weighting factors ( w1,w2 and w3). 


\section{DISTRIBUTYON SYSTEM SOFTWARE PROGRAMMING (DSSP)}

\subsection{Power and Energy Loss Computation in Medium Voltage( MV) Network}

The DSSP starts with closing all tie switches to create a meshed network. This meshed network will contain many closed loops, and each loop should has an optimal opening point for minimum losses. Therefore, our goal in normal operation is to find the optimal opening switch for each loop according to the following steps:

1. Input Data: which contains:

a) Feeder parameters (resistance and reactance), type of conductor, length and number of circuits.

b) Voltage, current, power factor, load factor and loss factor at the sending end of feeder.

c) Peak adjustment factor (P.A.F=Last Year Ann. Peak Load /Last Year Peak Load in the month corresponding to the month of measurements).

d) Node connection for each branch, crosssection area of each branch and percentage loading at each node.

2. Compute feeder input apparent power $(S=\sqrt{3} \mathrm{VI}$, where $V$ and $I$ are measured values.

3. Compute voltage profile, active power flow and reactive power flow in each section using $Z$-bus (based on measured transformers loading).

4. Compare feeder sending end calculated power with measured power within a certain tolerance $(\varepsilon \leq 0.0001$ for Gauss-seidel method).

5 . If (the measured power minus the calculated power) $\leq \varepsilon$ then stop calculations. Otherwise, modify the calculated power for each node according to the following relations:

$$
\begin{aligned}
& P_{\text {modiffed }}=\frac{S}{K V A_{\text {calculated }}} \times \text { input } K V A \text { loading } \times \cos \phi \\
& Q_{\text {modified }}=\frac{S}{K V A_{\text {calculated }}} \times \text { input } K V A \text { loading } \times \sin \phi
\end{aligned}
$$

6. Go to step 4 .

\subsection{Reconfiguration of Medium Voltage Loops Operation Schemes}

The medium voltage distribution system is designed as loops. These loops are normally opened under normal operating conditions.

The points of opening the loops are determined by the operation and control groups in the electricity distribution company.

This program presents a technique to determine the optimal operation scheme (point of opening) of the M.V. loops to minimize the power losses.

1. Input Data: which contains: a- Feeder sections data: type of conductor, length, number of circuits.

b- Actual voltage at the sending end.

c- Kiosks (transformer points) data (Active power, reactive load and capacity).

2- Computations

Based on the Medium Voltage cables and load data input, the program solves the load flow in the closed loop, and determines the optimal location of opening the loop to minimize the power losses. This location is the node at which the load flow reverses its direction.

The program calculates the load flow and power losses in the new operation system.

The reduction in power losses may be calculated by comparing the losses under original operating conditions with that in the optimal operating condition.

\section{PROPOSED OPTIMAL PROCEDURES}

The first optimal proposed procedure (POP) is concerned with the planning problem of finding the optimal cross-section areas of branches. The first branches in both sides of meshed system must have the capability to carry the total current in both distributors. In this procedure the MOGA is used to minimize the objective function (12) and satisfy the distribution system constraints (6)-(9). After the determination of the optimal cross-section areas of branches, the second POP is applied using the DSSP in order to find the best radial reconfiguration system to minimize the total system losses. However, a tie switch and some sectionalizing switches with the feeders form a loop. A certain switch of each loop is then selected to open to make the loop become radial, and the selected switch naturally becomes a tie switch. In the second POP, the network reconfiguration problem is identical to the problem of selection of an appropriate tie switch for each loop so that the power loss $\left(F_{1}\right)$ is minimized and the RDS operation and planning constraints (6)-(9) are satisfied.

\section{APPLICATIONS}

\subsection{Case Studies}

Two real cases distribution study are used to find the optimal planning and operation of these systems. These case studies are real parts of Tanta city in Egypt. Figures 3 and 4 show the configuration of these distribution systems and the input data are shown in Tables 1 and 2, respectively. These systems contain three normally open switches thatare closed only when a fault occurs.

Four cases are considered to select the optimal cross-section areas of the feeders which are:

In Case 1: the cross-section areas of all branches are equal to $150 \mathrm{~mm} 2$. 
In Case 2: the cross-section areas of all branches are equal to $150 \mathrm{~mm} 2$ except the first branches in both sides of meshed network for both distribution systems (1-2 \& 11-12 in the first system and 1-2 \& 24-25 in the second system) are equal to $240 \mathrm{~mm} 2$.

In Case 3: the cross-section areas of all branches are equal tol $50 \mathrm{~mm} 2$ except the two first branches in both sides of meshed network for both systems (1-3 \& 10-12 in the first system and 1-3 \& 23-25 in the second system) are equal to $240 \mathrm{~mm} 2$.

In Case 4: the cross-section areas of all branches are equal $150 \mathrm{~mm} 2$ except the three first branches in both sides of meshed network for both systems (1-4 \& 912 in the first system and 1-4 \& 22-25 in the second system) are equal to $240 \mathrm{~mm} 2$. However, the current capacities of cross-section areas $150 \mathrm{~mm} 2$ and 240 $\mathrm{mm} 2$ are equal to 219 and 289 Amperes, respectively.

Table 1 Input data for the first case study

\begin{tabular}{|c|c|c|c|c|}
\hline $\begin{array}{c}\text { Node } \\
\text { No. }\end{array}$ & $\begin{array}{c}\text { K.V.A. } \\
\text { rating }\end{array}$ & $\begin{array}{c}\text { Percentage } \\
\text { Loading\% }\end{array}$ & $\begin{array}{c}\text { Section } \\
\text { No. }\end{array}$ & $\begin{array}{c}\text { Length } \\
\text { m. }\end{array}$ \\
\hline 2 & 100 & 45 & 1 & 435 \\
\hline 3 & 500 & 50 & 2 & 75 \\
\hline 4 & 500 & 75 & 3 & 1000 \\
\hline 5 & 500 & 65 & 4 & 720 \\
\hline 6 & 500 & 75 & 5 & 300 \\
\hline 7 & 300 & 72 & 6 & 380 \\
\hline 8 & 500 & 64 & 7 & 120 \\
\hline 9 & 500 & 71 & 8 & 230 \\
\hline 10 & 250 & 48 & 9 & 540 \\
\hline 11 & 500 & 74 & 10 & 400 \\
\hline 12 & 200 & 52 & 11 & 140 \\
\hline Voltage level is 11KV, total current is 205 A, feeder loss \\
factor is 0.4013, power factor is 0.85 , and load Factor is 0.6046. \\
\hline
\end{tabular}

Table 2 Input data for the second case study

\begin{tabular}{|c|c|c|c|c|}
\hline $\begin{array}{c}\text { Node } \\
\text { No. }\end{array}$ & $\begin{array}{c}\text { K.V.A. } \\
\text { rating }\end{array}$ & $\begin{array}{c}\text { Percentage } \\
\text { Loading\% }\end{array}$ & $\begin{array}{c}\text { Section } \\
\text { No. }\end{array}$ & $\begin{array}{c}\text { Length } \\
\text { m. }\end{array}$ \\
\hline 2 & $0 .$. & 0. & 1 & 240 \\
\hline 3 & 300 & 64 & 2 & 270 \\
\hline 4 & 300 & 35 & 3 & 100 \\
\hline 5 & 1000 & 55 & 4 & 40 \\
\hline 6 & 500 & 51 & 5 & 350 \\
\hline 7 & 500 & 52 & 6 & 100 \\
\hline 8 & 500 & 44 & 7 & 100 \\
\hline 9 & 500 & 44 & 8 & 320 \\
\hline 10 & 500 & 56 & 9 & 200 \\
\hline 11 & 300 & 53 & 10 & 250 \\
\hline 12 & 1000 & 20 & 11 & 750 \\
\hline 13 & 100 & 60 & 12 & 100 \\
\hline 14 & 300 & 73 & 13 & 650 \\
\hline 15 & 160 & 39 & 14 & 30 \\
\hline 16 & 300 & 28 & 15 & 650 \\
\hline 17 & 300 & 47 & 16 & 50 \\
\hline 18 & 500 & 75 & 17 & 420 \\
\hline 19 & 500 & 71 & 18 & 150 \\
\hline 20 & 500 & 61 & 19 & 170 \\
\hline 21 & 250 & 75 & 20 & 380 \\
\hline 22 & 250 & 85 & 21 & 80 \\
\hline 23 & 250 & 65 & 22 & 400 \\
\hline 24 & 500 & 70 & 23 & 210 \\
\hline 25 & 300 & 37 & 24 & 70 \\
\hline Voltage level is 11 & KV, total current is 160 A, feeder loss factor is \\
\hline 0.4580, power factor is 0.93, and load Factor is 0.6220 \\
\hline
\end{tabular}

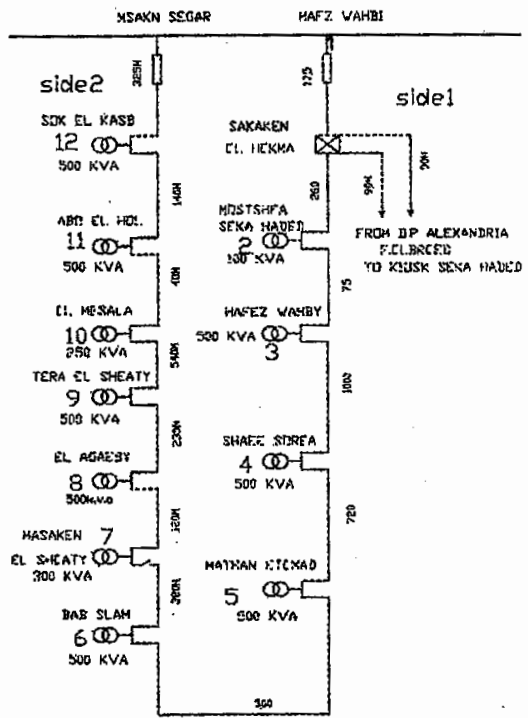

Fig. 3 Configuration of the first case study

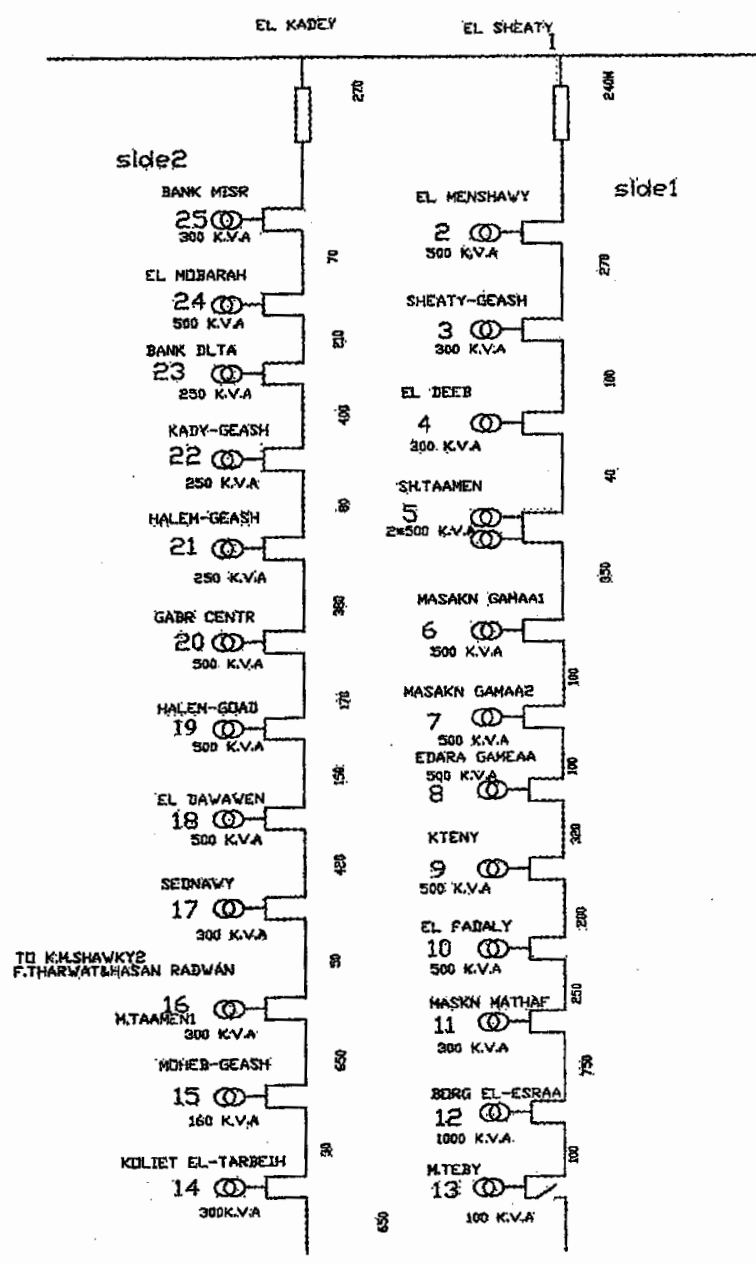

Fig. 4 Configuration of the first case study

Table 3 and 4 show the percentage increasing in load demand from year 2003 to year 2006 and the transformer rating as a KVA at each loading point for two case studies, respectively. From these tables, the increasing in load demand are varied from $35 \%$ to 
$77 \%$ and from $0 \%$ to $85 \%$ for two case studies, respectively. These tables are used to obtain the optimal planning of the distribution systems for the different cases (1-4).

Table 3 Percentage increasing in load demand for the first case study

\begin{tabular}{|c|c|c|c|c|c|}
\hline \multirow{2}{*}{ Node No. } & \multirow{2}{*}{ K.V.A. Rating } & \multicolumn{4}{|c|}{ Year } \\
\cline { 3 - 6 } & & 2003 & 2004 & 2005 & 2006 \\
\hline 2 & 100 & 35 & 51 & 51 & 45 \\
\hline 3 & 500 & 51 & 44 & 50 & 50 \\
\hline 4 & 500 & 59 & 65 & 66 & 75 \\
\hline 5 & 500 & 69 & 70 & 65 & 65 \\
\hline 6 & 500 & 76 & 77 & 75 & 75 \\
\hline 7 & 300 & 45 & 49 & 72 & 72 \\
\hline 8 & 500 & 61 & 63 & 64 & 64 \\
\hline 9 & 500 & 62 & 68 & 70 & 71 \\
\hline 10 & 250 & 40 & 43 & 48 & 48 \\
\hline 11 & 500 & 36 & 66 & 71 & 74 \\
\hline 12 & 500 & 51 & 52 & 54 & 52 \\
\hline
\end{tabular}

Table 4 Percentage increasing in load demand for the second case study

\begin{tabular}{|c|c|c|c|c|c|}
\hline \multirow{2}{*}{$\begin{array}{l}\text { Node } \\
\text { No. }\end{array}$} & \multirow{2}{*}{$\begin{array}{l}\text { K.V.A } \\
\text { Rating }\end{array}$} & \multicolumn{4}{|c|}{ Year } \\
\hline & & 2003 & 2004 & 2005 & 2006 \\
\hline 2 & 500 & 48 & 48 & 50 & 50 \\
\hline 3 & 300 & 67 & 57 & 71 & 64 \\
\hline 4 & 300 & 0 & 0 & 0 & 35 \\
\hline 5 & 1000 & 55 & 55 & 55 & 55 \\
\hline 6 & 500 & 44 & 44 & 51 & 51 \\
\hline 7 & 500 & 52 & 52 & 52 & 52 \\
\hline 8 & 500 & 39 & 43 & 41 & 44 \\
\hline 9 & 500 & 53 & 55 & 50 & 44 \\
\hline 10 & 500 & 53 & 53 & 56 & 56 \\
\hline 11 & 300 & 56 & 55 & 53 & 53 \\
\hline 12 & 1000 & 53 & 51 & 23 & 20 \\
\hline 13 & 100 & 56 & 56 & 60 & 60 \\
\hline 14 & 300 & 20 & 19 & 25 & $v_{r}$ \\
\hline 15 & 160 & 62 & 62 & 66 & 59 \\
\hline 16 & 300 & 53 & 40 & 63 & $\overline{Y A}$ \\
\hline 17 & 300 & 68 & 64 & 85 & $\xi Y$ \\
\hline 18 & 500 & 59 & 82 & 69 & $Y_{0}$ \\
\hline 19 & 500 & 51 & 54 & 55 & पi \\
\hline 20 & 500 & 69 & 65 & 73 & nा \\
\hline 21 & 250 & 68 & 63 & 73 & vo \\
\hline 22 & 250 & 10 & 33 & 43 & 10 \\
\hline 23 & 250 & 5 & 10 & 21 & 90 \\
\hline 24 & 500 & 32 & 42 & 65 & $Y$. \\
\hline 25 & 300 & 73 & 73 & 73 & $\overline{Y Y}$ \\
\hline
\end{tabular}

\subsection{Results and Comments}

Figures 5-8 show the optimal cross-section areas of the branches using the first POP (case 1) compared with other cases (2-4). The optimal cross-section areas of $150 \mathrm{~mm} 2$ is suitable for increasing in load demand in future (up to year 2009). While, Case 4 is more suitable for increasing in load demand up to year 2013. Figures 5 and 6 show the total costs (F1 and F2 ) of Equations (3) and (4) of cases (1-4) for each side of the distribution system using the first POP applied on the two case studies, respectively.

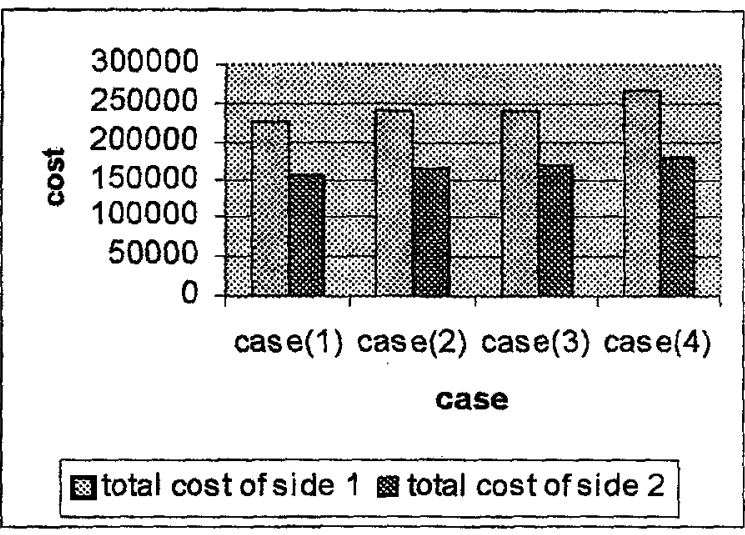

Fig. 5 Total costs of each side of the feeder using the first POP (case 1) compared with other cases (2-4) for the first case study.

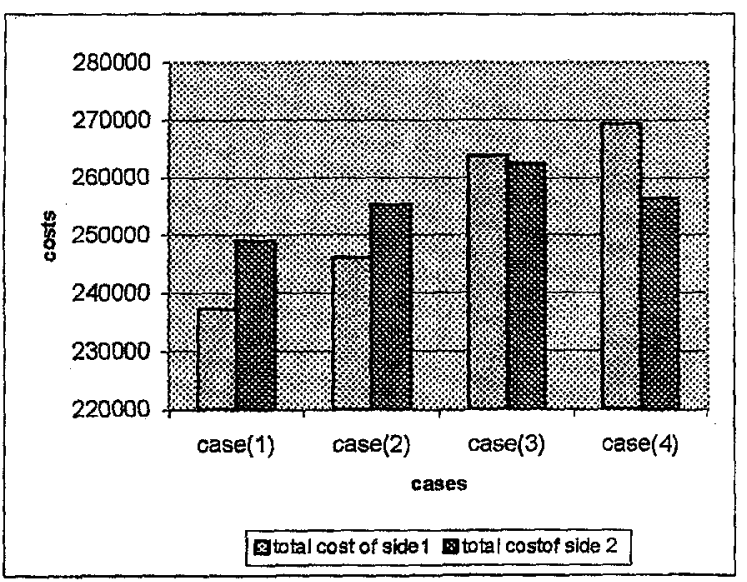

Fig.6 Total costs of each side of the feeder using the first POP (case 1) compared with other cases (2-4) for the second case study.

Figures 7 and 8 show the power losses (F1) in each side using the first POP for the two case studies, respectively. However, the minimization of power losses cost $F 1$ are very small compared with the feeder costs (F2) as shown in figure 9. Case 2 can be loaded in future up to $100 \%$ for the two case studies.

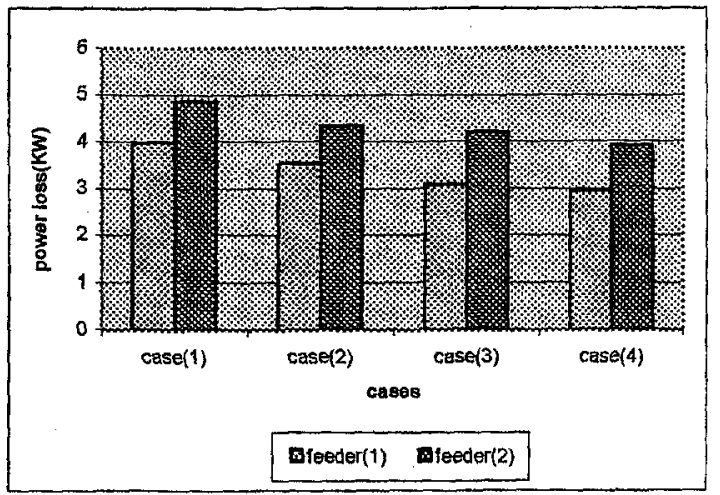

Fig. 7 Power losses in each side using the first POP (case 1) compared with other cases (2-4) for the first case study. 


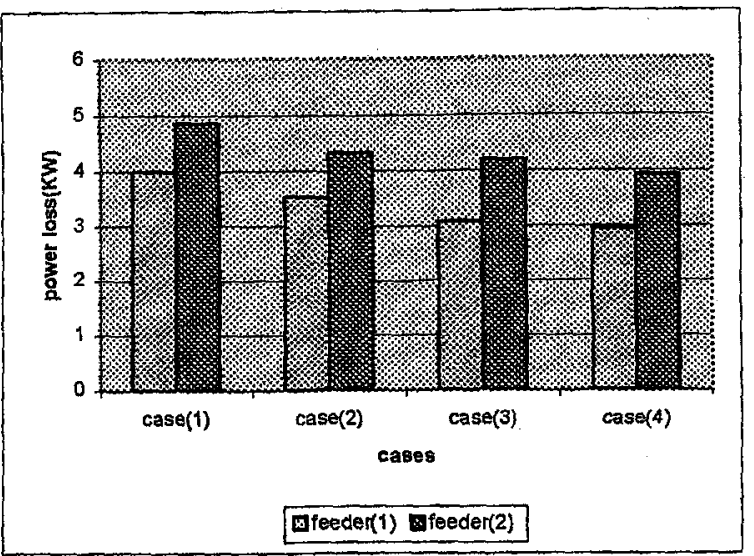

Fig.8 Power losses in each side using the first POP (case 1) compared with other cases (2-4) for the second case study.

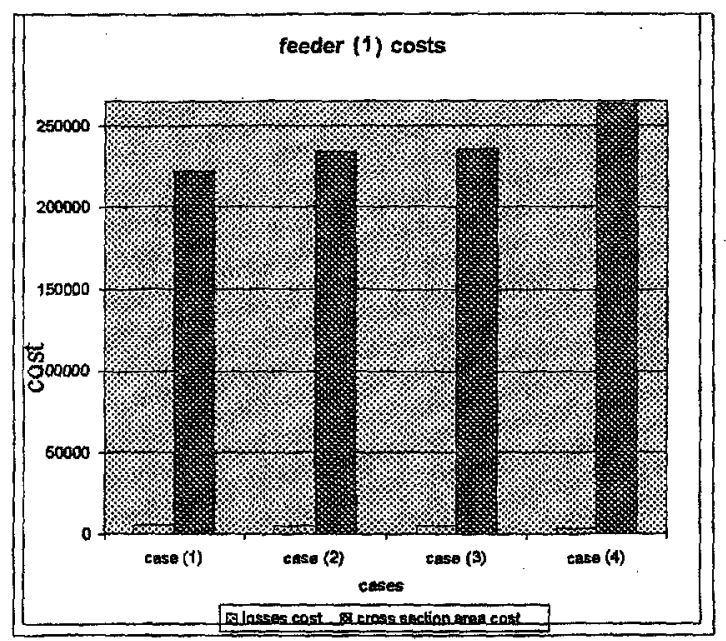

(a) First case study

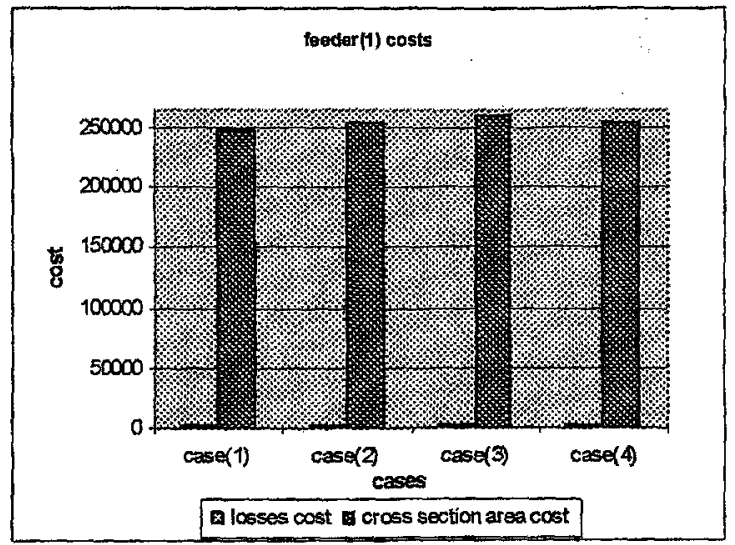

(b) second case study

Fig.9 Power losses and feeder costs using the first POP (case 1) compared with other cases $(2-4)$ for the two case studies.

Figures 10 and 11 show the voltage regulation factors for different cross-section areas (cases 1-4) applied on the first side of the RDS of the two case studies, respectively. From this figure, the voltage regulation factors are improved in the case 4 , but the total costs of power loss and feeder costs are increased compared with case1, as shown in Figures 5-8.

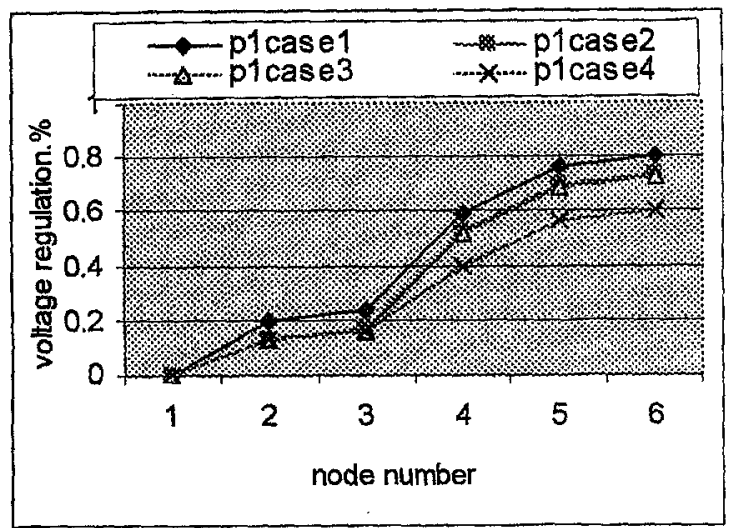

Fig.10 Voltage regulation factors for the first side of the first case study.

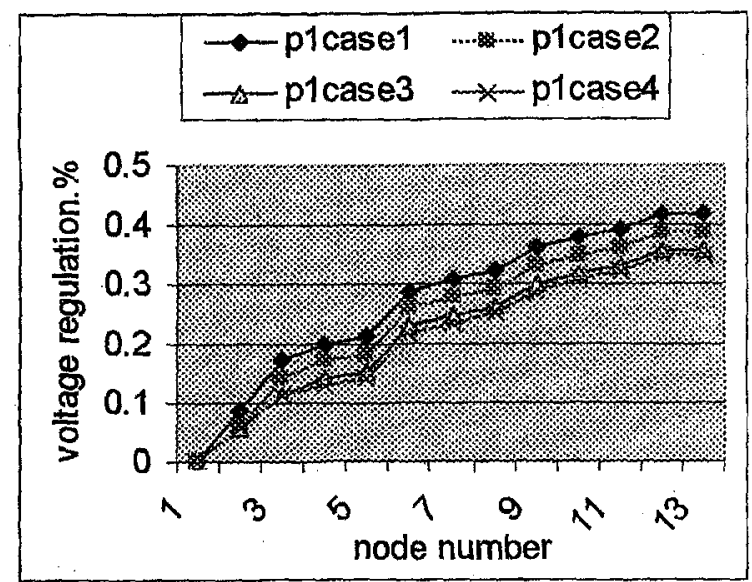

Fig.11 Voltage regulation factors for the first side of the second case study.

The second POP starts with closing all tie switches to create a meshed network. The tie switches are connected between nodes (4-5), (5-6) and (6-7) in the first case study, and between nodes (12-13), (13-14) and (14-15) in the second case study.

Table 5 A comparison between the three original tie switches for the first case study

\begin{tabular}{|c|c|c|c|}
\hline Variables & \multicolumn{3}{|c|}{ Original open switches } \\
\hline $\begin{array}{c}\text { Tie } \\
\text { switches }\end{array}$ & $5-6$ & $6-7$ & $7-8$ \\
\hline $\begin{array}{c}\text { Power } \\
\text { loss }(K W)\end{array}$ & 17.61657 & 16.3839 & 18.82705 \\
\hline $\begin{array}{c}\text { Voltage } \\
\text { Magnitude } \\
(K . V .)\end{array}$ & $\begin{array}{c}V_{\max }=11.00 \\
\text { at bus bar }\end{array}$ & $\begin{array}{c}V_{\max }=11.00 \\
\text { at bus bar }\end{array}$ & $\begin{array}{c}V_{\max }=11.00 \\
\text { at bus bar }\end{array}$ \\
\cline { 2 - 5 } & $V_{\min }=10.806$ & $V_{\min }=10.786$ & $V_{\min }=10.765$ \\
\hline
\end{tabular}

Tables 5 and 6 show the optimal opening switch to minimize the power loss while the voltage profile are maintain within the permissible limits using the second POP for the two case studies, respectively. From these tables, the minimum loss values are obtained using the second POP compared with the other original opening switches. However, the 
optimal open switches are between nodes 6-7 and 13.14 for the two case studies which have minimum power loss equal to $16.3839 \mathrm{KW}$ and $8.85491 \mathrm{KW}$, respectively.

Table 6 A comparison between the three original tie switches for the second case study.

\begin{tabular}{|c|c|c|c|}
\hline Variables & \multicolumn{3}{|c|}{ Original open switches } \\
\hline $\begin{array}{c}\text { Tie } \\
\text { switches }\end{array}$ & $12-13$ & $13-14$ & $14-15$ \\
\hline $\begin{array}{c}\text { Power } \\
\text { loss }(K W)\end{array}$ & 8.96233 & 8.85491 & 8.96428 \\
\hline $\begin{array}{c}\text { Voltage } \\
\text { Magnitude } \\
(K . V .)\end{array}$ & $\begin{array}{c}V_{\max }=11.00 \\
\text { at bus bar }\end{array}$ & $\begin{array}{c}V_{\max }=11.00 \\
\text { at bus bar }\end{array}$ & $\begin{array}{c}V_{\max }=11.00 \\
\text { at bus bar }\end{array}$ \\
\cline { 2 - 4 } & $V_{\min }=10.849$ & $V_{\min }=10.846$ & $V_{\min }=10.821$ \\
\hline
\end{tabular}

Similarly, comprehensive studies are carried out using the second POP to find the optimal opening switch for cases (1-4). From these studies, the optimal opening switches are independent on the changing of cross-section areas from case to another.

\section{CONCLUSION}

This paper presents an efficient proposed optimal procedures for planning and operation of distribution systems. The first procedure has been applied to find the optimal cross-section areas of branches in order to achieve multi-objective functions which are minimizing the power loss and feeder costs as well as minimization the voltage deviations with respect to the flat voltage (1.0 p.u.). The MOGA has been successfully applied to choice the optimal crosssection areas of the branches which are suitable for existing and expansion load demand while the distribution system constraints are satisfied. The second POP has been introduced for the distribution system reconfiguration to minimize power losses and restore service to the affected loads during the normal operating conditions. This problem is formulated and successfully solved using the DSSP to find the optimal opening switch in order to minimize the power losses and maintain the voltage profile within the permissible limits. Using the DSSP, it is noted that the optimal opening switch is independent of the size of branche's area and the effects of power loss costs are very small compared to the feeder costs. The optimal proposed procedures are more suitable for different sizes of practical network reconfiguration.

\section{REFERENCES}

[1] R. F. Sarfi, M. M. A. Salama, and A.Y. Chikhani, "A survey of the state of the art in distribution system reconfiguration for system loss reduction", Electr. Power Syst. Res., Vol. 31, pp. 61-70, 1994.

[2] A. B. Morton and I. M. Y. Mareels, "An efficient brute-force solution to the network reconfiguration problem", IEEE Trans. Power Del., Vol. 15 No.3, pp.996-1000, Jul. 2000.
[3] M. E. Baran and F. F. Wu, "Network reconfiguration in distribution systems for loss reduction and load balancing", IEEE Trans. Power Del., Vol. 4, No.2, pp. 1401- 1407, Apr. 1989.

[4] S. Civanlar et al., "Distribution feeder reconfiguration for loss reduction and service restoration", IEEE Trans. Power Del., Vol. 3, No.3, pp. 1217-1223, Jul. 1988.

[5] W.-M. Lin and H.-C. Chin, "Anew approach for distribution feeder reconfiguration for loss reduction and service restoration", IEEE Trans. Power Del., Vol. 13, No.3, pp. 870-875, Apr. 1998.

[6] Y.-J. Jeon, J.-C. Kim, J.-O. Kim, J.-R. Shin, and K. Y. Lee, "An efficient simulated annealing algorithm for network reconfiguration in largescale distribution systems", IEEE Trans. Power Del., Vol. 17, No.4, pp. 1070 - 1078, Oct. 2002.

[7] N. Kagan and C. C. de Barioni de Oliveira, "Fuzzy decision model for the reconfiguration of distribution networks using genetic algorithm", in Proc. 13th Power Syst. Comput. Conf., Vol. 17, Trondheim, Norway, pp. 929-935, Jun. 28-Jul. 2nd 1999.

[8] J. Z. Zhu, "Optimal reconfiguration of electrical disrtibution network using the genetic algorithm", Elect. Power Syst. Res., Vol.62, pp. 37-42, 2002.

[9] A. Augugliaro, L. Dusonchet, M. G. Ippolito, and E. R. Sanseverino, "Minimum losses reconfiguration of MV distribution networks through local control of tie- switches", IEEE Trans. Power Del., Vol. 18, No.3, pp.762 771, Jul. 2003.

[10] I.J.R.-Rosado, and J.L. Bernal- Agustin, "Reliability and Costs Optimization for Distribution Networks Expansion Using an Evolutionary Algorithm", IEEE Trans. on Power Syst., Vol. 16, No.1, pp. $111-118$, Feb. 2001.

[11] C. S. Chen, S.M.,M.S. Kang, J. C. Hwang, and C. W. Huang, "Temperature Effect to Distribution System Load Profiles and Feeder Losses", IEEE Trans. on Power Syst., Vol. 16, No.4, pp. 916 - 921, Nov. 2001.

[12] S. M. Ying-Yi Hong, and Saw-Yu Ho, "Determination of Network Configuration considering Multi objective in Distribution Systems Using Genetic Algorithms", IEEE Trans. On Power Syst,, Vol. 20, No.2, pp. 1062- 1069, May. 2005.

[13] H. P. Schmidt, N. Ida, Fellow, S. M., N. Kagan and J. C. Guaraldo, "Fast Reconfiguration of Distribution Systems Considering Loss Minimization", IEEE Trans. On Power Syst., Vol. 20, No.3, pp. 1311- 1318, Aug. 2005. 